\title{
Papers
}

\section{Novel consent process for research in dying patients unable to give consent}

\author{
Elizabeth Rees, Janet Hardy
}

\begin{abstract}
Objectives To develop a process of advance consent to enable research to be undertaken in patients in the terminal phase.

Design Feasibility study of an advance consent process to support a randomised controlled trial of two antimuscarinic drugs (hyoscine hydrobromide and glycopyrronium bromide) in the management of noisy respirations associated with retained secretions ("death rattle").

Setting Palliative care wards in a major cancer centre. Participants Patients admitted to a palliative care ward who may develop "death rattle" and thus be eligible for randomisation.

Main outcome measures Patient accrual; acceptability of the consent process.

Results Of the 107 patients approached, 58 patients gave advance consent to participate in the study. Of these, 15 patients developed death rattle and were randomised to receive either hyoscine or glycopyrronium; 16 patients died elsewhere; 15 patients died on the palliative care wards but were not randomised; 12 patients are still alive.

Conclusions Initial assessment suggests that this is a workable consent process allowing research to be undertaken in patients who are unable to give consent at the time of randomisation. Patient accrual rates to date are lower than needed to recruit adequate numbers in the time allotted to answer the research question.
\end{abstract}

\section{Introduction}

In order to participate in a clinical trial patients must receive, comprehend, and retain all the information necessary to allow them to give fully informed consent for that trial. ${ }^{1}$ Obtaining such consent is often very difficult in some disciplines, such as emergency medicine, elderly care, and palliative care. ${ }^{2-4}$

The European Union directive on good clinical practice in clinical trials (currently under review) may allow for a "legal representative" to consent on behalf of an incompetent adult. In the United Kingdom, however, no established means exists to obtain consent for the entry of patients into research studies when they are unable to give consent. This applies particularly to non-therapeutic research, where the principal intention is to extend knowledge to benefit future patients. ${ }^{5}$
By law, no one is able to give consent on behalf of an adult for treatment or research. Relatives or carers cannot be asked to give consent on behalf of a patient. Doctors cannot use the "best interest" principle as applies to the treatment of patients unable to give consent. ${ }^{1}$ Research has, on occasion, been undertaken on patients unable to give consent after a local ethics committee has agreed to it. ${ }^{6}$ In the United States, the Food and Drug Administration allows waiver of informed consent for emergency research as long as certain conditions are met. ${ }^{7}$ Considerable debate has taken place about this, ${ }^{8}$ and the ethics have been questioned. Many journals will no longer publish studies undertaken without consent. ${ }^{6}$

Patients in the terminal phase of their disease are prescribed a large number of drugs (often by unlicensed routes or for unlicensed indications) and subjected to various interventions, many of which are of unproved benefit. Terminally ill patients are often too unwell to be put through a lengthy process of information giving and consent. No reason exists why the management of dying patients should be excluded from the scientific scrutiny necessary for the treatment of patients in non-terminal situations. ${ }^{9}$ Research is essential to improve patient care and for palliative care to develop as an evidence based specialty. ${ }^{10}$

Although randomised controlled trials remain the gold standard, many people consider uncontrolled, observational, and qualitative studies to be more suited to research in palliative care. Whatever the research method, consent from patients is essential to ensure their protection and autonomy. ${ }^{11}$

\section{Background}

Dying patients are often unable to clear secretions from their large airways, resulting in noisy breathing usually described as "death rattle." This can be distressing to relatives and people caring for dying patients. Two antimuscarinic drugs are commonly used for the control of this condition. Hyoscine hydrobromide, a tertiary amine that can cross the blood-brain barrier causing central nervous system side effects, has historically been the drug of choice. Glycopyrronium bromide is a quaternary amine that does not cross the blood-brain barrier. Several audits and observational studies have indicated the efficacy of each of these drugs, but the range of reported response rates is wide (48-92\%). ${ }^{12}$ Only one controlled study has been carried out, in which 31 patients were randomised to receive
Department of Palliative Medicine, Royal Marsden Hospital, Sutton, Surrey SM2 5PT

Elizabeth Rees research nurse in palliative care Janet Hardy consultant in palliative care Correspondence to: J Hardy

bmj.com 2003;327:198

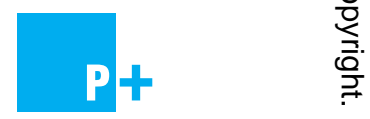

The patient information sheet is on bmj.com 
either hyoscine or placebo. ${ }^{13}$ The authors reported "tendentially" less death rattling in the active group. No consent was obtained for participation in this study.

Our aim was to undertake a study to assess the relative efficacy of hyoscine and glycopyrronium in the control of death rattle within the context of a randomised controlled trial. To do this, we needed a means of obtaining consent from patients who would be unable to give consent at the time of randomisation.

We presented the problem to our local ethics committee, which in turn consulted with medical ethicists and legal representatives. The ethics committee held a special meeting to discuss how such research could be carried out in dying patients. The consensus was that the development of an advance consent process was the only possible means of obtaining consent in this situation. This paper details a method of obtaining advance consent and the interim results of the recruitment process.

\section{Methods}

All patients admitted to the palliative care wards in the Royal Marsden Hospital are given an information sheet explaining that research is an integral part of patient care. The sheet details the types of studies undertaken on the palliative care unit and advises patients that they might be approached about research studies during their admission.

The "trial suitability" of patients is determined at pre-round multidisciplinary meetings. Patients too unwell, unable to understand English, or likely to be distressed are not approached.

At the next ward round, the consultant tells all potentially eligible patients of the "noisy breathing" study and asks them if they would be prepared to enter the study if they were ever to develop difficulty breathing because of retained secretions. The consultant reassures patients that this is not a problem that they have at the moment but one that may develop in the future. The term "death rattle" is not used unless prompted by the patient.

If patients are able to understand the preliminary information, are able to read the trial information, and express interest in the study, we give them the patient information sheet (see bmj.com). Relatives and carers are often involved in helping the patient to read and understand the information leaflet. The research nurse asks patients if they understand the study, answers any questions or concerns, and ascertains if they are willing to enter the study.

We then ask patients to sign the consent form in the presence of one of the study investigators. The research nurse, ward nurse, or the patient's relative or carer witnesses the consent. We reassure patients that they are free to change their mind at any stage and that refusal to enter the study will not prejudice their future care.

Once consent is obtained, glycopyrronium bromide and hyoscine hydrobromide are prescribed on the "as required" section of the patient's drug chart. The prescription is highlighted in red and labelled as a research study. The patient's agreement to enter the study is documented in the medical and nursing notes.

At each subsequent admission, we ask patients if they are still prepared to enter the study. We ask them to re-sign the consent form on each occasion. The prescription for antimuscarinic drugs can then be rewritten. If the patient is unable to re-sign the consent form, we ask the patient's relatives or carers if they know of any reason why the patient might have changed his or her mind about being in the study. Any concerns can then be discussed. If no indication exists that the patient had changed his or her mind, we consider the last consent to be valid and document it as such.

If the patient subsequently develops noisy breathing needing treatment, randomisation takes place. Nursing staff open a randomisation envelope that specifies which drug the patient is to receive and formally assess the response to the drug on a standard proforma. The research nurse runs a continuous education programme to ensure that all doctors and nurses on the ward are aware of the workings of the study.

Our statistician advised us to do an interim analysis to determine whether the consent process was working and if adequate numbers of patients were likely to be accrued for us to be able to assess the relative efficacy of the two drugs.

\section{Results}

In the seven month period from May to November 2002 we considered 107 patients for entry into the study (figure). Of these, 34 declined and 15 subsequently proved unable to consent. Fifty eight patients gave informed consent and were entered into the study. By January 2003, 15 of these patients had died on the palliative care wards but had not been randomised, because they did not develop death rattle, they died suddenly, or they were missed from the randomisation process. Sixteen patients died at home or in other units. Twelve patients are still alive. Five patients have reconsented once, and two patients have reconsented twice. We randomised 15 patients to receive either hyoscine or glycopyrronium at the time of death. We have not yet analysed the effectiveness or otherwise of the two agents in the patients recruited to date.

\section{Discussion}

We have previously considered the factors that influence participation of patients in clinical trials in

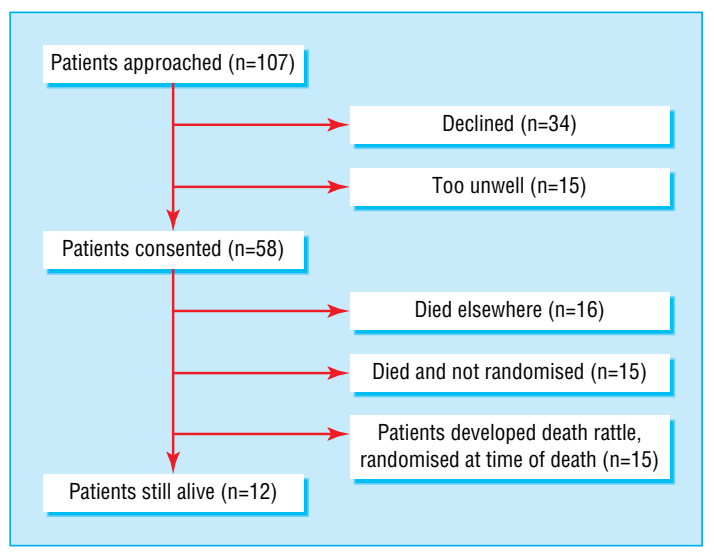

Patient flow in the study 
palliative care. ${ }^{14}$ Patients on palliative care wards are often of poor performance status and are too unwell to read lengthy patient information sheets. They are therefore unable to give consent. As a consequence, recruiting sufficient numbers of patients to complete studies is difficult. We developed this consent process in an attempt to improve recruitment of patients to trials in palliative care.

Assuming that the true rate of control of retained secretions at the time of death with both agents is about $60 \%$, we would need to recruit 250 patients to support the hypothesis that glycopyrronium is as effective as hyoscine in the management of death rattle. Approximately 200 patients die in the two palliative care wards at this hospital each year. We estimated that about half of these patients would develop death rattle. Therefore, we might hope to randomise 75-100 patients a year and to complete the study in three years.

Of the 58 patients who gave consent in a seven month period, we subsequently randomised 15 . Twelve patients have given consent and may be randomised in the future. We designed the study to include an interim analysis of the consent process, the recruitment rate, and the feasibility of completing the study in a single centre. Although the "process" is working well, we are unlikely to achieve our recruitment target without the participation of local hospices or other palliative care centres. Initial estimates of recruitment were overoptimistic. Many patients are too unwell even on their first admission to receive the information needed to give consent. More patients than expected were never readmitted to the unit and therefore never randomised despite having given consent.

The consent process is very time consuming and emotionally draining for staff. The success of the study depends on multidisciplinary input, both in screening suitable patients and in supporting those who have consented. A continuous education programme is essential to keep new doctors and nursing staff informed of the trial process.

Slight concern remains as to whether the patients consenting for this trial can ever be fully informed. Even if they have witnessed other patients with death rattle, they may not equate this with the condition being studied. Similarly, they may read the information less carefully if they consider the trial to be a distant event that may never happen. The ethics of "advance consent" for research in emergency medicine have been questioned. ${ }^{15}$

Very few patients have been distressed when approached about the study. The relatively high refusal rate indicates that patients feel free to decline entry if they are not willing to participate. The follow up visit by the research nurse after the consultant ward round allows patients the opportunity to decline entry in those cases where an initial acceptance might have been made only to "keep the doctor happy." Our records show that patients are much more likely to agree to enter a study when asked by a doctor than by a nurse.

Despite the fact that accrual has proved slower than anticipated, we are encouraged to continue by the success of the process so far. We believe that this is a workable means of obtaining consent for trials in terminally ill patients who cannot give consent. Our accrual figures to date indicate that we must involve other cen-

\section{What is already known on the topic}

Research to improve the evidence base behind the management of dying patients is very difficult

Patients in the terminal phase are given a large number of different drugs and subjected to many interventions of unproved benefit

Dying patients are usually too unwell to give informed consent for trials

What this study adds

This study presents a consent process that allows patients to consent in advance to a trial for which they may be eligible at a later date

If accepted, this process has the potential to facilitate research in the care of dying patients

tres in order to recruit the patient numbers necessary to answer the research question. The complexity of the randomisation and assessment process would make the current study very difficult to undertake by palliative care teams caring for patients in their own homes.

Research in palliative care is notoriously difficult. ${ }^{16}$ If generally accepted, this consent process may provide a means of increasing the number of patients in the terminal phase entering trials. This is essential if we are to improve the evidence base underpinning the practice of palliative care and improve the care of dying patients.

We thank the nursing and junior medical staff on Horder and Chevallier Wards at the Royal Marsden Hospital; J Riley and K Broadley for recruiting and consenting patients; research nurses B Gwilliam and A Kennett; Roger A'Hern for statistical advice; and the ethics committee of the Royal Marsden Hospital.

Contributors: JH and ER developed the study and wrote the paper. JH will act as guarantor.

Funding: ER is funded by the Royal Marsden Palliative Care Research Fund.

Competing interests: None declared.

Ethical approval: The scientific committee and the ethics committee of the Royal Marsden Trust approved the study.

1 Department of Health. www.doh.gov.uk/consent (accessed Nov 2002). Biros MH, Lewis RJ, Olson CM, Runge JW, Cummins RO, Fost N. Informed consent in emergency research. JAMA 1995;273:1283-7.

Informed consent in emergency research.JAMA 1995;273:1283-7.
Warren JW, Sobal J, Tenney JH, Hoopes JM, Damron D, Levenson S, et al. Informed consent by proxy. N Engl J Med 1986;315:1124-8.

Hardy J. Consent for trials in palliative care. Lancet Perspectives 2000;356:s44.

5 Assessment of mental capacity: guidance for doctors and lawyers: a report of the British Medical Association and the Law Society.London: BMA, 1995.

Smith R. Informed consent: the intricacies. BMI 1997;314:1059-60.

7 Adams JG, Wegener J. Acting without asking: an ethical analysis of the Food and Drug Administration waiver of informed consent for emergency research. Ann Emerg Med 1999;33:218-23.

8 Doval L, Tobias JS, Warnock M, Power L, Goodare H. Informed consent Doyal L, Tobias JS, Warnock M, Power L, Good
in medical research. $B M J$ 1998:316:1000-5.

9 Hardy J. Placebo controlled trials in palliative care-the argument for. Hardy J. Placebo controlled
Palliat Med 1997;11:415-8.

Palliat Med 1997;11:415-8.
Hermet R, Burucoa B, Sentilhes-Monkam A. The need for evidencebased proof in palliative care. EurJ Palliat Care 2002;9:104-7.

11 Worthington R. Clinical issues on consent: some philosophical concerns. J Med Ethics 2002;28:377-80.

12 Bennett M, Lucas V, Brennan M, Hughes A, O'Donnell V, Wee B. Using antimuscarinic drugs in the management of death rattle: evidence-based guidelines for palliative care. Palliat Med 2002;16:369-74.

13 Likar R, Molnar M, Rupacher E, Pipman W, Deutsch J, Mortl M, et al. A clinical randomised double-blind study comparing the effectiveness of scopolamine hydrobromide with placebo in patients with death rattle [abstract]. 7th European Association of Palliative Care Conference, [abstract]. 7th 
14 Ling J, Rees E, Hardy J. What influences participation in clinical trials in palliative care in a cancer centre? Eur J Cancer 2000;36:621-6.

The letter or the spirit: consent for research in CPR. JAMA 1994:271:1445-7.
16 Grande GE, Todd CJ. Why are trials in palliative care so difficult? Palliat Med 2000;14:69-74.

(Accepted 14 May 2003) 